\title{
WHO mulls phase out of vaccine linked to polio outbreaks
}

$\mathrm{T}$ he World Health Organization (WHO) is contemplating discontinuing the use of an oral polio vaccine that's been linked to ongoing outbreaks of the virus in the developing world.

WHO is weighing the possibility of using a different formulation of the vaccine, but maintains that patchy implementation of immunization programs, not the vaccine itself, is to blame for the outbreaks.

Since 2000, some two billion children have received more than 10 billion doses of oral polio vaccine containing up to three live strains of the virus, says Oliver Rosenbauer, spokesperson for the WHO's Global Polio Eradication Initiative.

Nineteen outbreaks of circulating vaccine-derived poliovirus (cVDPV) have been documented in that same period, resulting in 536 cases in some 17 countries, the majority in Africa, Rosenbauer says.

One outbreak in Nigeria has been ongoing since 2005, with 13 new cases reported so far this year (www.polio eradication.org/Dataandmonitoring /Poliothisweek/Circulatingvaccine derivedpoliovirus.aspx).

While less virulent than wild poliovirus, and therefore more easily controlled, cVDPV is just as harmful, Rosenbauer says. "Once wild poliovirus eradication has been achieved, the use of oral polio vaccine in routine immunization will need to be stopped, and work is already underway to prepare for this."

Outbreaks of cVDPV occur when routine immunization activities are poorly conducted and a population is left susceptible to poliovirus, whether wild or vaccine-derived, Rosenbauer explains. "The problem is not the vaccine itself, but low vaccination coverage. If a population is fully immunized, they will be protected against both vaccine-derived and wild polioviruses."

More than $90 \%$ of all cVDPV cases stem from the mutation of just one of

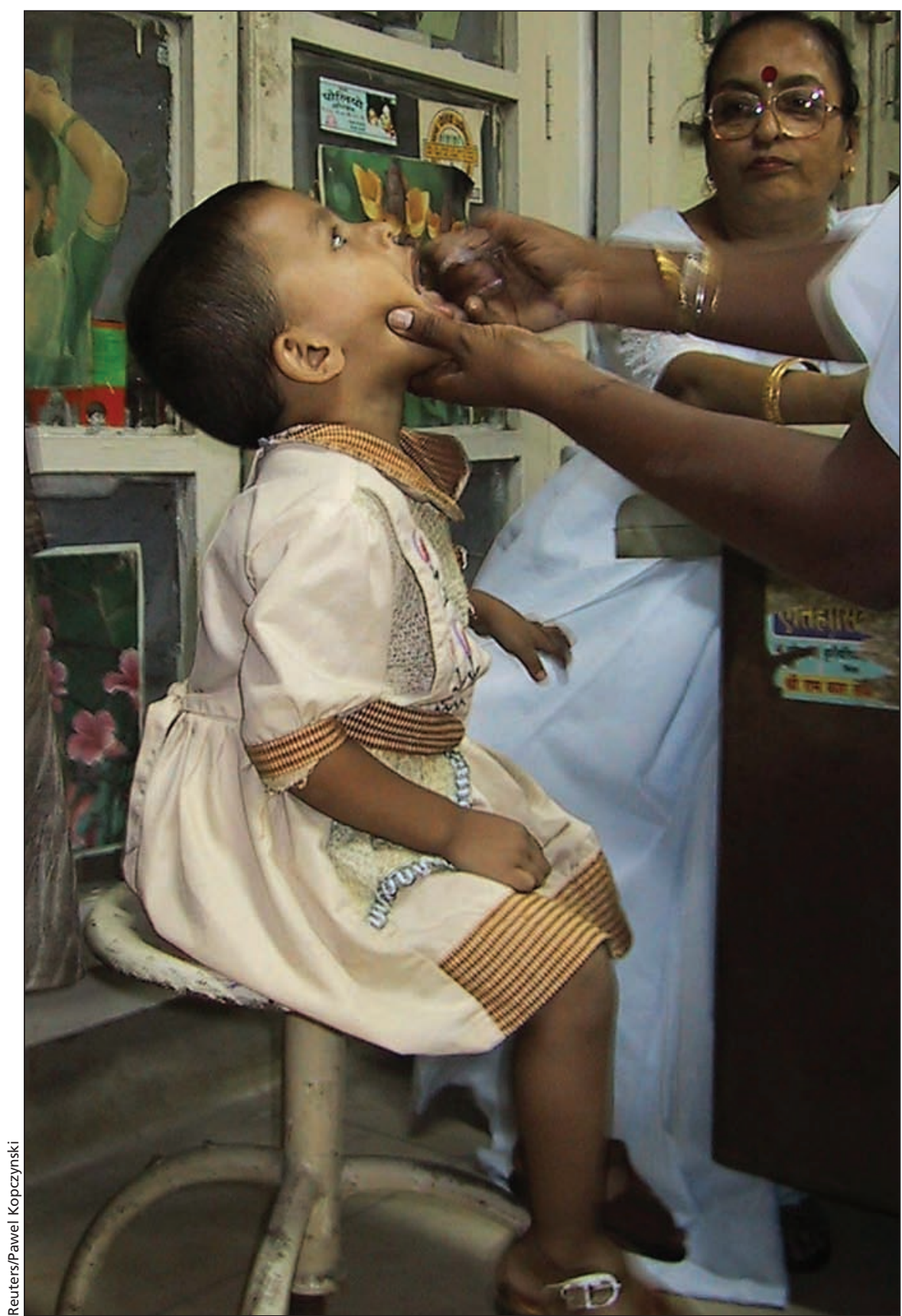

A young girl receives oral polio vaccine from a doctor in India.

the three live polio strains in the vaccine, type 2 vaccine-virus, says Rosenbauer. "However, wild poliovirus type 2 has already been globally interrupted since 1999."

Inactivated polio vaccine, routinely used in the developed world, isn't prac- tical for use in polio-infected countries because it only offers individual immunity, Rosenbauer adds.

However, given the availability of a new oral polio vaccine containing type 1 and 3 virus-strains only, "there is a suggestion to switch from trivalent OPV 
[oral polio vaccine] to bivalent OPV at this time already, even as efforts continue to eradicate type 1 and 3 wild poliovirus," he says.

Scaling up use of the new oral vaccine has the potential to accelerate the eradication of type 1 and 3 wild polioviruses as well as greatly reduce the risk for new cVDPV cases when global surveillance and response capacity is highest, Rosenbauer explains.

But the bivalent oral vaccine could not be used to immunize against transmission of cVDPV type 2. That creates a catch-22 situation for countries with existing outbreaks as they'd have to rely on the same vaccines that cause the outbreaks to curb them.

In countries affected by all three serotypes - wild type 1, wild type 3 and cVDPV type 2 - getting the vaccine mix right is critical, Rosenbauer says. In Nigeria, a combination of monovalent, bivalent and trivalent oral polio vaccines continues to be used to boost immunity to all three serotypes, he adds.

"For success to be achieved, we need the full engagement of the politi- cal leadership in countries which are polio-infected to fully implement [immunization] activities," Rosenbauer says. "The feasibility of poliovirus eradication has been demonstrated in India, where, as a result of full application [of WHO-recommended strategies], no case has been reported since January 2011."

Cost evaluations of phasing out trivalent oral polio vaccine use are ongoing. - Lauren Vogel, CMAJ

CMAJ 2011. DOI:10.1503/cmaj.109-4027 